\title{
Caracterización de sistemas agroecológicos para el establecimiento comercial de cacao orgánico (Theobroma cacao) en Talamanca
}

\author{
Description of agro-ecological systems \\ to the shop of organic cocoa \\ (Theobroma cacao) in Talamanca
}

Ricardo Salazar Díaz 


\section{Resumen}

Un requisito previo para la planificación de una finca es disponer de información acerca de las características del productor, o productora, y su familia, así como de los recursos naturales disponibles y el sistema de producción que tiene, ya que, para proponer cambios en el uso y manejo de los recursos naturales, hay que conocer, analizar e interpretar lo que se dispone en la unidad productiva.

Es por esto que se planteó caracterizar cinco fincas ubicadas en el territorio indígena Bribri en la comunidad de Shuabb, distrito Telire, cantón de Talamanca, Limón, Costa Rica, para la producción de cacao orgánico (Theobroma cacao) bajo un sistema tradicional agroforestal.

El enfoque de producción orgánica con un manejo sostenible de los recursos naturales coincide con la norma tradicional y espiritual de los indígenas.

Se estableció una tipología basada en aspectos biofísicos para las cinco fincas seleccionadas y una caracterización socioeconómica de las familias beneficiarias; con base en los resultados obtenidos, se propuso un diseño de producción y un manejo agronómico orgánico para el establecimiento de los sistemas agroecológicos.

\section{Palabras clave}

Cacao orgánico, caracterización socioeconómica, tipificación biofísica, indígenas.

\section{Abstract}

A prerequisite for planning a farm is to have available information about the characteristics of the producer and her or his family, about the available natural resources and about the existing production system. For proposing changes in the use and management natural resources, is necessary to know, to analyze and interpret what is available in the production unit.

This is why it is plated characterize five farms located in an indigenous community named Shuabb, in Telire district, Talamanca, Limón, Costa Rica. For the organic cocoa (Theobroma cacao) production, under a traditional agroforestry system.

The organic approach under a sustainable management of natural resources, coincides with the traditional and spiritual standard of the indigenous.

A typology was established based on biophysical aspects for the five selected farms and a socioeconomic characterization of the beneficiary families; based on the results it was proposed a production design and an agronomic management, for establishing the agroecological systems.

\section{Key words}

Organic cocoa, socioeconomic and biophysical characterization, indigenous. 


\section{Introducción}

El cantón de Talamanca, en Costa Rica, engloba los territorios indígenas más extensos y más poblados del país, los cuales cuentan con un régimen administrativo autónomo, sustentado por leyes y convenios internacionales. Por diversas circunstancias históricas, Talamanca ha tenido poca participación en el proceso de desarrollo del país, lo cual ha determinado que, en la actualidad, exista un evidente retraso en su desarrollo con respecto a otras áreas rurales o urbanas (Borge y Castillo, 1997).

El cacao (Theobroma cacao) es realmente apreciado al ser un fruto nativo que ha alimentado la historia, cultura y economía de los pueblos indígenas de Mesoamérica. En la actualidad, Talamanca es una de las principales zonas productoras de cacao en Costa Rica; además, el cacao está retomando una participación importante en el sector agrícola del país, después de haber sufrido una caída importante en la producción nacional en los años ochenta.

Se requiere mejorar la producción sostenible del cacaotal para incrementar los ingresos de los productores y evitar la expansión de cultivos menos diversos y pobremente estructurados (por ejemplo, banano y plátano) que reduzcan el potencial de los territorios indígenas para conservar la biodiversidad (Somarriba y Harvey, 2003).

La caracterización está dirigida fundamentalmente a cuantificar las variables que tipifican una zona determinada, con el fin de establecer la vocación, posibilidades y limitaciones de sus recursos naturales y las condiciones socioeconómicas de las comunidades que la habitan. La caracterización debe de ser interpretativa, en el sentido de identificar que puede darse entre las distintas variables (Jiménez, 2005).

A partir de la tipificación particular de cada una de las fincas seleccionadas en esta investigación, se obtiene la información necesaria para determinar la viabilidad técnica para la producción comercial de cacao orgánico, respetando el conocimiento ecológico indígena.

\section{Materiales y métodos}

Es necesario establecer que este estudio es de tipo secuencial o cronológico, ya que permite estudiar la realidad desde los acontecimientos presentes para darles seguimiento en un futuro inmediato, que consistirá en el establecimiento de sistemas agroecológicos productivos.

\section{Descripción del área de estudio}

El estudio se realizó en el poblado indígena Bribri, Shuabb, distrito Telire del cantón de Talamanca, que se ubica en el extremo sureste de la provincia de Limón, Costa Rica. Este poblado se encuentra dentro del Parque Internacional La Amistad, reconocido por la UNESCO como Sitio de Patrimonio Mundial de la Humanidad debido a la gran importancia y diversidad de ecosistemas que protege. Su ubicación geográfica está entre los $8^{\circ} 37^{\prime}$ y 9 9०38' de latitud norte, y entre los $82^{\circ} 24^{\prime}$ y $83^{\circ} 25^{\prime}$ de longitud oeste. La zona de vida corresponde al Bosque Húmedo Tropical (bh-T), la precipitación promedio anual es de 2000-2400 mm y tiene una temperatura media anual de $24-26^{\circ} \mathrm{C}$ (Bolaños et al. 2005).

\section{Caracterización socioeconómica}

Se identificaron las características más sobresalientes de la economía y de aspectos sociales de las familias, las cuales servirán para conocer y proponer soluciones para el cambio y desarrollo de los sistemas de producción.

Como fuente secundaria de información, se recurrió al Programa de Asistentes Técnicos de Atención Primaria del EBAIS de Bambú, que es el centro médico correspondiente para los pobladores de Shuabb.

\section{Selección de las parcelas de estudio}

Las propietarias de las cinco fincas seleccionadas fueron: Maribel Iglesias López, Carlota Torres Trejos, Yorley Oniel Morales, Tany Romero Yaslyn, Nodia Rodríguez Almengor. Estas fincas fueron seleccionadas a partir de los siguientes criterios: ser productoras agropecuarias; tener a disposición parcelas de, al menos, media hectárea de terreno cada una para la realización de la investigación; colaborar con la mano de obra para realizar las labores de campo; tener, las propietarias, disponibilidad de ser entrevistadas; y permitir la visita a sus fincas. 
Cuadro I. Resultados de aspectos socioeconómicos de las beneficiarias

\begin{tabular}{|l|c|c|l|l|c|}
\hline \multicolumn{1}{|c|}{ Finca } & $\begin{array}{c}\text { Tamaño de la } \\
\text { propiedad (ha) }\end{array}$ & $\begin{array}{c}\text { Tenencia de la } \\
\text { tierra }\end{array}$ & Lugar donde vive & $\begin{array}{c}\text { Mano de obra } \\
\text { familiar }\end{array}$ & $\begin{array}{c}\text { Uso actual de la } \\
\text { tierra }\end{array}$ \\
\hline Maribel Iglesias & 1 & Propia & En la finca & $\begin{array}{l}\text { Ella y su } \\
\text { compañero }\end{array}$ & Charral \\
\hline Nodia Rodríguez & 2 & Propia & En la finca & $\begin{array}{l}\text { Ella y su } \\
\text { compañero }\end{array}$ & Tacotal \\
\hline Tany Romero & 2 & Propia & Afuera de la finca & Ella & Charral \\
\hline Yorley Oniel & 2,5 & Propia & Afuera de la finca & $\begin{array}{l}\text { Ella y dos } \\
\text { hermanos }\end{array}$ & Tacotal \\
\hline CarlotaTorres & 2 & Propia & Afuera de la finca & Ella y su hijo & Arrozal \\
\hline
\end{tabular}

Fuente: Elaboración propia.

\section{Caracterización de las parcelas}

Se caracterizaron las parcelas por ubicación, altura (msnm), topografía del terreno, porcentaje de pendiente (plana 0-5\%; ondulada 5-15\%; quebrada mayor de 15\%), dirección de la pendiente y porcentaje de sombra.

\section{Muestreo y análisis de suelos}

El muestreo de suelos se realizó tomando en cada finca una muestra compuesta de 10 puntos aleatorios a una profundidad de $0-40 \mathrm{~cm}$. A cada muestra se le determinó la textura, la densidad aparente y se le realizó un análisis químico completo, $C$ (materia orgánica) y N. Los análisis se llevaron a cabo en el laboratorio de suelos y de recursos naturales del Centro de Investigaciones Agronómicas de la Facultad de Ciencias Agroalimentarias de la Universidad de Costa Rica. También se hicieron muestras de suelos a $20 \mathrm{~cm}$, para determinar el color del suelo con la Tabla Munsell.

\section{Elaboración del diseño de las fincas}

Con respecto al material genético del cacao, se optó por recomendar seis clones seleccionados por el Centro Agronómico Tropical de Investigación y Enseñanza (CATIE), que han demostrado, en resultados preliminares, un comportamiento óptimo (rendimiento de producción y tolerancia a enfermedades) bajo las condiciones de la región; tres de ellos autocompatibles (CATIE R-I CC-I 37 e ICS-95) y tres autoincompatibles (CATIE R-4, CATIE R-6 y PMCT-58). Se incluyó un sétimo clon, IMC-67, como clon donante de polen, que también se utiliza como porta injerto. Además, se recomienda incluir otros cuatro clones para la producción de semilla porta injerto (UF-6 I3, SPA-9, EET-400 y PA- I69).

\section{Resultados y discusión}

\section{Caracterización socioeconómica de las familias beneficiarias}

Estas son familias de escasos recursos económicos que cuentan con una casa propia, pero solo una de las familias posee electricidad. Los principales problemas que comparten son el desempleo y que sus únicos ingresos son por medio de la agricultura, la mayoría de subsistencia debido al problema de la comercialización. Por otra parte, la situación escolar se basa en que, al terminar la primaria, no continúan los estudios secundarios.

\section{Diagnóstico biofísico de las parcelas}

Las medidas de temperatura, realizadas puntualmente en las cinco parcelas, oscilan entre 30$35^{\circ} \mathrm{C}$, resultados relativamente altos ya que, en la región, la temperatura media anual es de $24-26{ }^{\circ} \mathrm{C}$ (MAG-CCT, 2000). Estas mediciones en campo se realizaron durante un medio día de verano, lo cual podría explicar que estén por arriba del promedio anual.

En cuanto a la cantidad de lluvia en la zona, la precipitación promedio anual es de 2000-2400 mm (MAG-CCT, 2000), por lo que es satisfactoria para el cultivo de cacao.

Los resultados de las mediciones de la velocidad del viento, realizadas puntualmente en las cinco parcelas, fueron de 0,0 m/seg., que pareciera favorecer los bajos índices de caída de hojas prematuras, ocasionada por la intensidad del viento. 
Cuadro 2. Resultados de factores biofísicos de las parcelas en estudio

\begin{tabular}{|l|c|c|c|c|c|c|c|}
\hline \multicolumn{1}{|c|}{ Parcela } & $\mathrm{T}^{\circ} \mathrm{C}$ & Alt & \%HR & Ms & \% pend & Dir. pend & \% sombra \\
\hline Maribel Iglesias & 32,2 & 80 & 63,5 & 0,0 & 32 & $50^{\circ}$ N.E & 10 \\
\hline Nodia Rodríguez & 32,1 & 94 & 67,4 & 0,0 & 30 & $285^{\circ}$ N.O & 50 \\
\hline Tany Romero & 34,7 & 64 & 52,2 & 0,0 & 2 & --- & 10 \\
\hline Yorley Oniel & 32,8 & 175 & 63,6 & 0,0 & 40 & $5^{\circ}$ N.E & 50 \\
\hline CarlotaTorres & 30 & 224 & 67,4 & 0,0 & 35 & $30^{\circ}$ N.E & 2 \\
\hline
\end{tabular}

Fuente: Elaboración propia.

Al ser débil la velocidad del viento, los árboles de sombra defenderán adecuadamente al cacao para que no sufra daños. Por lo tanto, no va ser necesario recomendar establecer cortinas rompe vientos. Asimismo, en todas las parcelas hay bosques cercanos que favorecen este factor.

Por su parte, la humedad relativa no es un factor que pueda equipararse en importancia a los factores anteriormente analizados, aunque también puede contribuir a la propagación de algunas enfermedades. Podríamos decir que es un factor que no presenta condiciones especiales que puedan constituir una limitante.

\section{Descripción de los suelos de estudio}

\section{Textura}

La parcela de Maribel Iglesias presenta un suelo de textura franca, el promedio de arcillas resultó ser bajo (23\%). De acuerdo con el manual de conservación de suelos de ladera citado por Núñez (1985), se indica que este tipo de suelo tiene una buena aireación y buena infiltración, además de tener una consistencia ligeramente adhesiva y una capacidad media de retención de humedad.
La parcela de Tany Romero presenta un suelo de textura franco arcilloso; este tipo de suelo tiene una infiltración y aireación regular, además tiene una consistencia adhesiva y una capacidad media de retención de humedad.

Las parcelas de Nodia Rodríguez, Yorley Oniel y Carlota Torres presentan un suelo de textura fina arcillosa, con un promedio de arcillas alto (43\%, $70 \%$ y $58 \%$, respectivamente); este tipo de suelos presenta una infiltración deficiente y una aireación muy pobre, además, su consistencia es muy adhesiva y su capacidad de retención de humedad es muy alta.

\section{Densidad}

La densidad aparente de los suelos ronda el $1 \mathrm{gr} / \mathrm{cm}^{3}$, lo que sugiere suelos con poca intervención humana y que no han sido mecanizados, además de un buen desarrollo radical y facilidad en la penetración de las raíces.

\section{Contenido de materia orgánica}

Con respecto a estos resultados, se recomienda hacer de nuevo el análisis de suelo de materia orgánica con muestras de suelos obtenidas a $15 \mathrm{~cm}$ de profundidad, y no a $40 \mathrm{~cm}$ como se hizo, para poder obtener un mejor parámetro de interpretación.

Cuadro 3. Análisis de textura de los suelos de estudio

\begin{tabular}{|l|c|c|c|c|}
\hline & & Granulometría) & & Clase \\
\hline ID Usuario & Arena & Limo & Arcilla & Textural \\
\hline Maribel & 45 & 32 & 23 & Franco \\
\hline Yorley & 10 & 20 & 70 & Arcilloso \\
\hline Tany & 35 & 35 & 30 & Franco Arcilloso \\
\hline Carlota & 18 & 24 & 58 & Arcilloso \\
\hline Nodia & 30 & 27 & 43 & Arcilloso \\
\hline
\end{tabular}


Cuadro 4. Análisis de densidad de los suelos en estudio

\begin{tabular}{|l|c|c|c|}
\hline & Densidad aparente & Densidad de partículas & $\%$ porosidad \\
\hline ID usuario & $\mathrm{g} \mathrm{cm}^{-3}$ & $\mathrm{~g} \mathrm{~cm}^{-3}$ & \\
\hline Maribel & 1,27 & 2,57 & 50,85 \\
\hline Yorley & 0,80 & 2,61 & 69,35 \\
\hline Tany & 0,94 & 2,69 & 65,06 \\
\hline Nodia & 1,03 & 3,00 & 65,67 \\
\hline
\end{tabular}

Fuente: Laboratorio de recursos naturales CIA, UCR.

Cuadro 5. Análisis de carbono, nitrógeno y materia orgánica de los suelos en estudio

\begin{tabular}{|l|c|c|c|c|}
\hline & \multicolumn{2}{|c|}{$\%$} & $\begin{array}{c}\text { Relación } \\
\%\end{array}$ & \\
\hline ID usuario & $\mathrm{C}$ & $\mathrm{N}$ & $\mathrm{C} / \mathrm{N}$ & $\mathrm{MO}$ \\
\hline Maribel & 1,46 & 0,16 & 9,1 & 2,09 \\
\hline Yorley & 1,06 & 0,13 & 8,2 & 1,52 \\
\hline Tany & 1,62 & 0,17 & 9,5 & 2,32 \\
\hline Carlota & 1,63 & 0,20 & 8,2 & 2,33 \\
\hline Nodia & 1,20 & 0,13 & 9,2 & 1,72 \\
\hline
\end{tabular}

Fuente: Laboratorio de suelos y foliares CIA, UCR.

Cuadro 6. Resultados de la medición de los colores de los suelos en estudio con la Tabla Munsell

\begin{tabular}{|c|c|c|c|}
\hline Parcela & HUE & Value/Chroma & Color \\
\hline Maribel & 7,5 YR & $3 / 4$ & Café oscuro \\
\hline Nodia & 7,5 YR & $4 / 4$ & Café \\
\hline Tany & 7,5 YR & $4 / 3$ & Café \\
\hline Yorley & 7,5 YR & $5 / 8$ & Café fuerte \\
\hline Carlota & 7,5 YR & $3 / 4$ & Café oscuro \\
\hline
\end{tabular}

Fuente: Elaboración propia.

Cuadro 7. Análisis químico de los suelos en estudio

\begin{tabular}{|l|c|c|c|c|c|c|c|c|c|c|c|c|}
\hline \multicolumn{1}{|c|}{ Solución Extractora: } & $\mathrm{pH}$ & \multicolumn{4}{|c|}{$\mathrm{cmol}(+) / \mathrm{L}$} & $\%$ & \multicolumn{4}{c|}{$\mathrm{mg} / \mathrm{L}$} \\
\hline KCl-Olsen Modificado & $\mathrm{H}_{2} \mathrm{O}$ & $\mathrm{ACIDEZ}$ & $\mathrm{Ca}$ & $\mathrm{Mg}$ & $\mathrm{K}$ & $\mathrm{ClCE}$ & $\mathrm{SA}$ & $\mathrm{P}$ & $\mathrm{Zn}$ & $\mathrm{Cu}$ & $\mathrm{Fe}$ & $\mathrm{Mn}$ \\
\hline ID usuario & 5,5 & 0,5 & 4 & $\mathrm{I}$ & 0,2 & 5 & & 10 & 3 & $\mathrm{I}$ & 10 & 5 \\
\hline Maribel & 6,1 & 0,16 & 20,40 & 5,69 & 0,46 & 26,71 & 0,6 & $\mathrm{I}$ & 2,0 & $\mathrm{I}$ & 16 & 24 \\
\hline Yorley & 4,9 & 19,79 & 6,77 & 2,40 & 0,19 & 29,15 & 68 & $\mathrm{ND}$ & 5,1 & 8 & 154 & 17 \\
\hline Tany & 5,9 & 0,27 & 21,78 & 5,15 & 0,21 & 27,41 & $\mathrm{I}$ & 2 & 2,9 & 3 & 35 & 11 \\
\hline Carlota & 4,9 & 11,08 & 10,58 & 4,05 & 0,26 & 25,97 & 43 & $\mathrm{ND}$ & 3,4 & 10 & 134 & 66 \\
\hline Nodia & 5,8 & 0,54 & 20,19 & 7,50 & 0,69 & 28,92 & 2 & $\mathrm{ND}$ & 2,6 & 5 & 25 & 16 \\
\hline
\end{tabular}

Fuente: Laboratorio de suelos y foliares CIA, UCR. 
Cuadro 8. Relación entre bases, expresada en cmol (+)/L

\begin{tabular}{|l|c|c|c|c|}
\hline \multicolumn{5}{|c|}{ Relaciones de bases } \\
\hline Muestra & $\mathrm{Ca} / \mathrm{Mg}$ & $\mathrm{Mg} / \mathrm{K}$ & $\mathrm{Ca}+\mathrm{Mg} / \mathrm{K}$ & $\mathrm{Ca} / \mathrm{K}$ \\
\hline Rango aceptado & $2-5$ & $2,5-15$ & $10-40$ & $5-25$ \\
\hline Maribel & 4 & 12 & 57 & 44 \\
\hline Nodia & 3 & 11 & 40 & 29 \\
\hline Tany & 4 & 25 & 128 & 104 \\
\hline Yorley & 3 & 13 & 48 & 36 \\
\hline Carlota & 3 & 16 & 56 & 41 \\
\hline
\end{tabular}

Fuente: Elaboración propia.

Normalmente, el contenido de materia orgánica es alto en los primeros centímetros de suelo y disminuye en profundidad. Esto se define como una disminución regular del contenido de carbono orgánico.

\section{Color del suelo}

En general, todos comparten el color café, lo que se podría interpretar como suelos con presencia de materia orgánica.

\section{Propiedades químicas}

En lo que respecta al elemento fósforo, en las muestras de Yorley, Carlota y Nodia, el elemento no fue detectado, pero en las muestras de Tany y Maribel se encontraron $2 \mathrm{mg} / \mathrm{L}$, y I mg/L, respectivamente. Cabe destacar que valores menores a $10 \mathrm{mg} / \mathrm{L}$ son considerados deficientes de acuerdo con la tabla de niveles críticos (Bertsch, 1987).

Por lo tanto, esto nos lleva a plantear una recomendación general para todos los suelos, que sería aplicar roca fosfórica como enmienda natural del microelemento fósforo. La fórmula de la roca fosfórica comercial es $\mathrm{P}_{2} \mathrm{O}_{5}$, que equivale a un $18 \%$ de fósforo. Si se considera que hay un 30\% de deficiencia por fijación con otros elementos, se recomienda aplicar de 50-40 Kg/ha de $\mathrm{P}_{2} \mathrm{O}_{5 ;}$ si se divide esta cantidad por mil árboles de cacao por hectárea, para aplicarla de forma localizada, se obtiene una cantidad de 50-40 gramos de $\mathrm{P}_{2} \mathrm{O}_{5}$ por árbol.

En el caso del zinc, el cobre, y el manganeso, los valores de todas las muestras se encuentran dentro del rango apropiado para cada elemento. En el caso del hierro, las muestras de los suelos de Maribel, Tany y Nodia se encuentran dentro del rango apropiado, pero las muestras de los suelos de Yorley y Carlota sobrepasan el rango establecido en $54 \mathrm{mg} / \mathrm{L}$ y 34 $\mathrm{mg} / \mathrm{L}$, respectivamente, aunque a estos niveles no representan ningún riesgo en la nutrición de las plantas.

\section{Parcela de Maribel}

El $\mathrm{pH}$ presenta niveles favorables para el cultivo $(6, I)$. En lo que respecta al contenido de acidez, el valor es de $0,16 \mathrm{cmol}(+) / L$, lo que establece un nivel bajo de acidez, favorable también para el cultivo. El porcentaje de saturación de acidez está muy por debajo del $10 \%$ (0,6\%), propicio para cualquier cultivo.

Con respecto a los cationes cambiables, o bases (Ca, Mg, K), se puede decir que un suelo puede presentar una abundante cantidad de cada catión $y$, sin embargo, ofrecer malas posibilidades de absorción de los mismos para la planta debido a que se encuentran en proporciones desequilibradas. En este caso, la cantidad de cationes de potasio $(0,46$ $\mathrm{cmol}(+) / L)$ se encuentra entre los valores medios y, por lo tanto, es favorable para las plantas. En lo que respecta al calcio y magnesio, los valores son de 20,40 y $5,69 \mathrm{cmol}(+) / L$, respectivamente, lo cual supera el límite del rango medio, que es de 4-20 cmol (+)L para el calcio y I-5 cmol (+)/L para el magnesio, por consiguiente, se ubica en la categoría alta.

Al interpretar el cuadro 8, se presentan dos situaciones: las relaciones $\mathrm{Ca} / \mathrm{Mg}$ y $\mathrm{Mg} / \mathrm{K}$ se encuentran dentro del rango aceptado, a diferencia de las relaciones $\mathrm{Ca}+\mathrm{Mg} / \mathrm{K}$ y $\mathrm{Ca} / \mathrm{K}$, que son más 


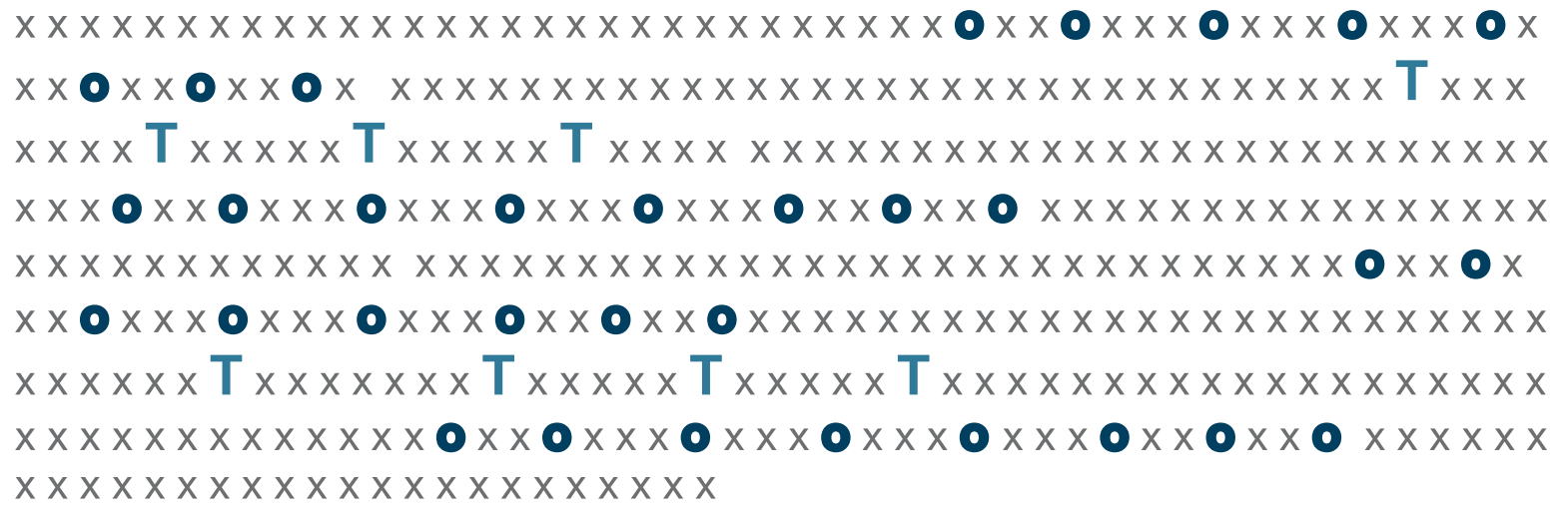

Figura I. Propuesta de diseño de siembra para media hectárea de cacao.

Fuente: Elaboración propia.

altas que el rango aceptado. Esto puede significar que hay un exceso de calcio respecto al potasio. La recomendación en este sentido es aumentar el potasio, que podría hacerse mediante la aplicación de fertilizantes orgánicos ricos en potasio.

\section{Parcela de Nodia}

El pH presenta niveles favorables para el cultivo $(5,8)$. En lo que respecta al contenido de acidez, el valor es de $0,54 \mathrm{cmol}(+) / L$, lo que establece un nivel bajo de acidez, favorable para el cultivo. El porcentaje de saturación de acidez está por debajo del I0\% (I\%), propicio para el cultivo.

En lo que respecta al calcio, magnesio y potasio, los valores son 20,40, 5,69 y 0,69 cmol (+)/L respectivamente. Estas cantidades superan el límite del rango medio, el cual es $4-20 \mathrm{cmol}(+) / L$ para el calcio, I-5 cmol (+)/L para el magnesio y 0,2-0,6 $\mathrm{cmol}(+) / L$ para el potasio, por consiguiente, se ubica en la categoría alta.

Al interpretar el cuadro 8, se presenta una situación muy favorable, pues todas las relaciones $(\mathrm{Ca} / \mathrm{Mg}$ $\mathrm{Mg} / \mathrm{K}, \mathrm{Ca}+\mathrm{Mg} / \mathrm{K}$ y $\mathrm{Ca} / \mathrm{K}$ ) se encuentran dentro del rango aceptado.

\section{Parcela de Tany}

El $\mathrm{pH}$ presenta niveles favorables para el cultivo $(5,9)$. En lo que respecta al contenido de acidez, el valor es de $0,27 \mathrm{cmol}(+) / L$, lo que establece un nivel dentro del rango aceptado de acidez, favorable para el cultivo. El porcentaje de saturación de acidez está por debajo del $10 \%$ (2\%), propicio para el cultivo.

En este caso, la cantidad de cationes de potasio $(0,2$ I $\mathrm{cmol}(+) / L)$ se encuentra entre los valores medios, y, por lo tanto, es favorable para las plantas. En lo que respecta al calcio $(21,78 \mathrm{cmol}(+) / L)$ y magnesio $(5,15 \mathrm{cmol}(+) / \mathrm{L})$, la cantidad obtenida supera el límite del rango medio, el cual es $4-20 \mathrm{cmol}(+) / L$ y $1-5 \mathrm{cmol}(+) / L$ respectivamente, por consiguiente, se ubica en la categoría alta.

Al interpretar el cuadro 8, se presentan dos situaciones: la relación $\mathrm{Ca} / \mathrm{Mg}$ se ubica dentro del rango aceptado, a diferencia de las relaciones $\mathrm{Mg} / \mathrm{K}, \mathrm{Ca}+\mathrm{Mg} / \mathrm{K}$ y $\mathrm{Ca} / \mathrm{K}$, que son mucho más altas que el rango aceptado. Esto puede significar que hay una deficiencia de potasio respecto al calcio y el magnesio; en otras palabras, existe un exceso de calcio y de magnesio respecto al potasio. La recomendación en este sentido es aumentar el potasio, que podría hacerse mediante la aplicación de fertilizantes orgánicos ricos en este elemento.

\section{Parcela de Yorley}

El pH presenta niveles desfavorables para el cultivo $(4,9)$. En lo que respecta al contenido de acidez, el valor es de 19,79 $\mathrm{cmol}(+) / L$; lo que establece un 
nivel muy alto de acidez, desfavorable también para el cultivo.

Con respecto a los cationes cambiables o bases ( $\mathrm{Ca}$, $\mathrm{Mg}, \mathrm{K}$ ), se puede decir que la cantidad de cationes de potasio, magnesio y calcio, con valores de 0,19, 2,40 y $6,77 \mathrm{cmol}(+) / L$ respectivamente, se encuentra entre los valores medios, lo que es favorable para las plantas.

Al interpretar el cuadro 8, se presentan dos situaciones: las relaciones $\mathrm{Ca} / \mathrm{Mg}, \mathrm{Mg} / \mathrm{K}$ y $\mathrm{Ca} / \mathrm{K}$ se encuentran dentro del rango aceptado, a diferencia de las relaciones $\mathrm{Ca}+\mathrm{Mg} / \mathrm{K}$ que son más altas que el rango aceptado. Esto significa que hay una deficiencia de potasio respecto al calcio más magnesio.

\section{Parcela de Carlota}

El pH presenta niveles desfavorables para el cultivo $(4,9)$. En lo que respecta al contenido de acidez, el valor es de II,08 cmol (+)/L; lo que establece un nivel muy alto de acidez, desfavorable también para el cultivo. El porcentaje de saturación de acidez está muy por encima del I0\% (43\%), nocivo para el cultivo.

Con respecto a los cationes cambiables o bases ( $\mathrm{Ca}$, $\mathrm{Mg}, \mathrm{K})$, se puede decir que la cantidad de cationes de potasio, magnesio y calcio, con valores de 0,26, 4,05 y $10,58 \mathrm{cmol}(+) / L$ respectivamente, se encuentra entre los valores medios, por lo tanto es favorable para las plantas.

Al interpretar el cuadro 8, se presentan dos situaciones; la relación Ca/Mg se encuentra dentro del rango aceptado, a diferencia de las relaciones $\mathrm{Mg} / \mathrm{K}, \mathrm{Ca}+\mathrm{Mg} / \mathrm{K}$ y $\mathrm{Ca} / \mathrm{K}$ que son ligeramente más altas.

En general, químicamente, el suelo de la parcela de Yorley y Carlota tiene problemas de acidez intercambiable y un porcentaje de saturación de acidez muy alto, lo que, probablemente, implica mucho contenido de aluminio libre, que es tóxico para las plantas. En este sentido, para el desarrollo de cualquier actividad agrícola, resulta apropiada la aplicación de una enmienda permitida por la certificación orgánica de cal (Ca), a una dosis localizada de 100 gramos por arbolito.

\section{Propuesta del diseño de siembra en el campo de cacao}

\section{Siembra en el campo}

En primer lugar, se deben seleccionar las mejores plantas del vivero. Un día antes del trasplante, lo mejor es hacer un buen riego a los viveros. Se debe tener listo el terreno, el cual debe estar limpio de malezas dañinas y protegido con malezas amigables.

\section{Distancia de siembra}

Se recomienda una distancia de $2 \mathrm{~m} \times 4 \mathrm{~m}$, con lo que se obtiene I 250 plantas por hectárea; esto favorece la aplicación de muchas prácticas en forma más fácil.

\section{Diseño de distribución de los clones}

Los seis clones seleccionados por el CATIE son intercompatibles, es decir, que se polinizan entre ellos; por lo anterior, y para facilidad, la siembra se puede hacer en filas, alternando en cada fila un clon. Si se utiliza este sistema de siembra, es posible mantener la identificación de los clones.

Otra forma de siembra puede ser aleatoria, es decir, sembrar plantas mezcladas de cada clon, siempre teniendo presente que debe haber una buena mezcla de plantas.

\section{Sombra para el cacao}

Para la sombra temporal, se puede usar bananos y plátanos, que se deben sembrar de cuatro a siete meses de anticipación a la plantación del cacao; también se puede sembrar yuca y maíz tres meses antes a la plantación de cacao. Estos cultivos cumplirán su objetivo de protección, y luego su cosecha dará suficientes ingresos para pagar buena parte de los gastos de establecimiento del cacao, ya que permanecerán durante los primeros tres a cinco años.

La sombra permanente está constituida por árboles que son más altos que el cacao. Por tradición, se ha elegido el árbol de guaba (Inga sp), ya que, por sus propiedades agroecológicas, aporta nitrógeno al suelo, provee de frutos comestibles y su madera es usada tradicionalmente para leña. Se recomienda sembrarla a una distancia de $3 \mathrm{~m} \times 3 \mathrm{~m}$ hasta llegar a una densidad de $24 \mathrm{~m} \times 24 \mathrm{~m}$ en el primer año. 
Existe la posibilidad de escoger especies maderables como el laurel blanco (Cordia alliodora), laurel negro (C. gerascanthus o C. megalantha) y el cedro (Cedrela $s p$.$) . El laurel se puede sembrar a una distancia de$ $12 \mathrm{~m} \times 12 \mathrm{~m}$, mientras que el cedro a $14 \mathrm{~m} \times 14 \mathrm{~m}$.

La figura I muestra una propuesta de diseño para el establecimiento de un sistema agroforestal de media hectárea, aunque, si bien es cierto, las parcelas en estas fincas no son exactamente rectangulares (ver anexos (-5); lo importante es definir las distancias de siembra que, para el cacao, es de $2 \mathrm{~m} \times 4 \mathrm{~m}$, para las guabas es de $24 \mathrm{~m} \times 24 \mathrm{~m}$, y para los laureles, de 12 $\mathrm{m} \times 12 \mathrm{~m}$. Los cultivos de sombra temporal, como los bananos y los plátanos, se deben sembrar a una distancia de $3 \mathrm{~m} \times 3 \mathrm{~m}$ entre las filas del cacao; la yuca y el maíz se deben sembrar a distancias cortas en la misma fila del cacao.

\section{Conclusiones}

Se estableció una tipología basada en aspectos biofísicos para las fincas de las cinco beneficiarias. Las cinco fincas presentan condiciones biológicas y meteorológicas óptimas para el desarrollo del cultivo de cacao.

Edafológicamente, las cinco fincas son aptas para el cultivo de cacao, sin embargo, hay algunas diferencias químicas entre ellas. La finca de Nodia tiene un suelo ideal para el cultivo, mientras que las fincas de Maribel y de Tany tienen una deficiencia de potasio que vendría a ser provocado por un desbalance por el exceso de calcio. Las fincas de Yorley y Carlota tienen problemas de acidez intercambiable y un porcentaje de saturación de acidez muy alto, que implica mucho contenido de aluminio libre, el cual es tóxico para las plantas. En este sentido, para el desarrollo del cultivo, es apropiada la aplicación de una enmienda permitida por la certificación orgánica de cal (Ca), a una dosis localizada de 100 gramos por arbolito sembrado.

Se logró recopilar información relevante para realizar y proponer un diseño de siembra, el cual fue aceptado, por parte de las cinco beneficiarias, para el establecimiento del cultivo.

\section{Bibliografía}

Bertsch, F. (1987). Manual para interpretar la fertilidad de los suelos de Costa Rica. ( $2^{\circ}$ ed.). San José: Universidad de Costa Rica.

Bolaños, R.; Watson, V \& Tosi, J. (2005). Mapa ecológico de Costa Rica (Zonas de Vida), según el sistema de clasificación de zonas de vida del mundo de L.R Holdridge, Escala 1:750:000. Centro Científico Tropical, San José, Costa Rica.

Borge, C. \& Castillo, R. (1997). Cultura y conservación en la Talamanca indígena. (pp. I30- I50). San José: EUNED.

Jiménez, F. (2005). Curso maestría manejo de cuencas hidrográficas. CATIE. Turrialba, Costa Rica

Nuñez, J. (1985). Fundamentos de edafología. ( $2^{\circ}$ ed.). (pp 6|-68). San José: EUNED.

Somarriba, E \& Harvey, C. (2003). ¿Cómo integrar producción sostenible y conservación de biodiversidad en cacaotales orgánicos indígenas? Agroforestería en las Américas, 10: 37-38. 